\title{
Two-dimensional superharmonic stability of finite- amplitude waves in plane Poiseuille flow
}

\author{
By J. D. PUGH AND P. G. SAFFMAN \\ Applied Mathematics, California Institute of Technology 217-50, Pasadena, CA 91125, USA
}

(Received 28 May 1987 and in revised form 8 December 1987)

In recent work on shear-flow instability, the tacit assumption has been made that the two-dimensional stability of finite-amplitudes waves in plane Poiseuille flow follows a simple and well-understood pattern, namely one with a stability transition at the limit point in Reynolds number. Using numerical stability calculations we show that the application of heuristic arguments in support of this assumption has been in error, and that a much richer picture of bifurcations to quasi-periodic flows can arise from considering the two-dimensional superharmonic stability of such a shear flow.

\section{Introduction}

We are concerned with the two-dimensional stability of two-dimensional steady waves (secondary flows) in plane Poiseuille flow (PPF), to infinitesimal perturbations of the same wavelength as the secondary flow (i.e. superharmonic disturbances). The secondary flows have been computed by a number of authors in recent years (e.g. Zahn et al. 1974; Herbert 1976; Milinazzo \& Saffman 1985) and stability analyses of them have focused on the three-dimensional stability problem. One reason is because three-dimensional perturbations grow on a convective timescale as opposed to the slower growth of two-dimensional disturbances (Orszag \& Patera 1983).

Nevertheless, the two-dimensional superharmonic stability problem is not without interest. In figure 1 we sketch a cross-section (at a representative streamwise wavenumber $\alpha$ ) of the two-dimensional secondary flow surface, with a characteristic amplitude plotted against a characteristic Reynolds number for the flow. Orszag \& Patera (1981) have used a one-dimensional phase representation of the energy to predict that the lower branch of the two-dimensional solution curve is unstable and the upper branch is stable to two-dimensional superharmonic disturbances. This stability transition (by which we mean where $\max _{\sigma} \operatorname{Re}(\sigma)=0, \sigma$ being the eigenvalue of the stability problem) at the 'nose' or limit point in Reynolds number is illustrated in figure 1. They admitted that their argument in support of this prediction is oversimplified and we shall demonstrate by numerical examples that it is not in general correct. In the rest of $\S 1$ we discuss the calculation of the two-dimensional secondary flows and in $\$ 2$ formulate the two-dimensional stability problem for these flows. There exist neutrally stable eigenmodes (i.e. with $\sigma=0$ ) at the nose, whose existence will be proved in $\$ 3$, but as shown by the numerical results presented in $\$ 4$ these coexist with unstable modes. Further, we shall demonstrate that there are bifurcations to quasi-periodic flows on the upper branch of the solution curve of figure 1.

$\uparrow$ Details are not given in their paper, and we have been unable to reproduce the argument in any rigorous fashion. 


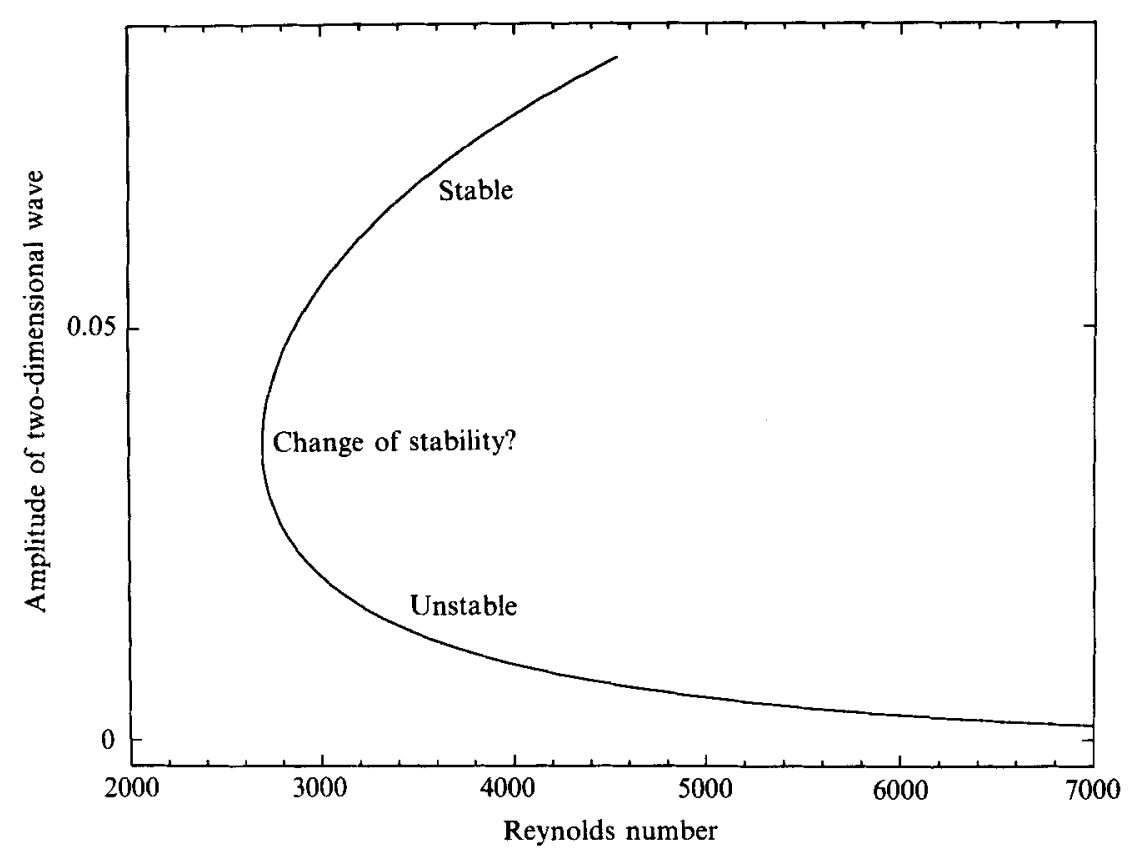

FIgURe 1. Naïve prediction of stability transition on a two-dimensional secondary flow solution curve. The figure is sketched for a wavenumber for which PPF is linearly stable.

The picture of stability transition in figure 1 is complicated by the lack of uniqueness in the parameterization of the two-dimensional flows. Two possible choices are a flux Reynolds number $\left(R e_{Q}\right)$ and a pressure Reynolds number $\left(R e_{P}\right)$. To understand how these arise and how they are related to one another we must consider the problem of calculating the two-dimensional secondary flows.

\subsection{Calculation of two-dimensional steady waves}

Consider the incompressible two-dimensional Navier-Stokes equations written in stream-function formulation:

$$
\frac{\partial \nabla^{2} \tilde{\Psi}}{\partial t}+\tilde{\Psi}_{y} \nabla^{2} \tilde{\Psi}_{x}=\tilde{\Psi}_{x} \nabla^{2} \tilde{\Psi}_{y}+\nu \nabla^{4} \tilde{\Psi}
$$

where the tildes denote dimensional quantities.

We look for steady, periodic travelling waves moving down the channel $-h \leqslant y \leqslant h$ with wave speed $c$ in the streamwise direction $x$. With the transforma-

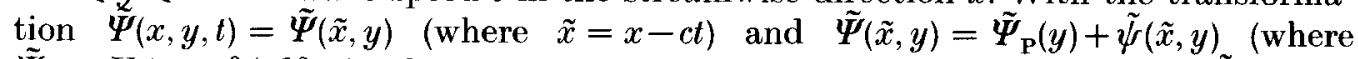
$\tilde{\Psi}_{\mathrm{P}}=U_{0}\left(y-y^{3} / 3 h^{2}\right)$ is the stream-function of the uniform PPF, and $\tilde{\psi}$ is the perturbation of the disturbed flow away from this basic flow), and also nondimensionalizing by the channel half-width $h$ and the characteristic velocity $U_{0}$, we have the perturbation equation

$$
-\frac{1}{R e} \nabla^{4} \psi+\left(\Psi_{\mathrm{P}, y}-c\right) \nabla^{2} \psi_{x}-\psi_{x} \Psi_{\mathrm{P}, y y y}+\psi_{y} \nabla^{2} \psi_{x}-\psi_{x} \nabla^{2} \psi_{y}=0
$$

where $R e=h U_{0} / \nu$ and for convenience we now write $x$ for $\tilde{x}$. We solve this by spectrally decomposing $(2)$ in the $x$-direction, writing

$$
\psi(x, y)=\sum_{n=-\infty}^{+\infty} \hat{\psi}_{n}(y) \mathrm{e}^{\mathrm{i} \alpha n x}
$$


Note that $\hat{\psi}_{0}$ is a perturbation to the uniform PPF. For reality of $\psi(x, y)$ we require

$$
\hat{\psi}_{-n}(y)=\hat{\psi}_{n}^{*}(y)
$$

which allows us to solve only for the non-negative modes, $n \geqslant 0$.

The modal equations corresponding to (2) for mode $n$ are

$$
\begin{array}{r}
-\frac{1}{R e}\left(S_{x}^{4}+2 S_{x}^{2} \frac{\mathrm{d}^{2}}{\mathrm{~d} y^{2}}+\frac{\mathrm{d}^{4}}{\mathrm{~d} y^{4}}\right) \hat{\psi}_{n}+\left(\Psi_{\mathrm{P}, y}-c\right)\left(S_{x}^{2}+\frac{\mathrm{d}^{2}}{\mathrm{~d} y^{2}}\right) S_{x} \hat{\psi}_{n}-\Psi_{\mathrm{P}, y y y} S_{x} \hat{\psi}_{n} \\
+\hat{\psi}_{y} *\left(S_{x}^{2}+\frac{\mathrm{d}^{2}}{\mathrm{~d} y^{2}}\right) S_{x} \hat{\psi}-\left(S_{x} \hat{\psi}\right) *\left(S_{x}^{2}+\frac{\mathrm{d}^{2}}{\mathrm{~d} y^{2}}\right) \hat{\psi}=0
\end{array}
$$

where $S_{x} f_{n}=\mathrm{i} \alpha n f_{n}$ and the convolution $f * g$ for mode $n$ is defined :

$$
f * g=\sum_{q=-\infty}^{+\infty} f_{n-q} g_{q} .
$$

The corresponding modal boundary conditions deduced from no-slip at the channel walls are

$$
\left.\begin{array}{rrr}
n>0: & \hat{\psi}_{n, y}( \pm 1)=0 & \left(u_{n}( \pm 1)=0\right), \\
\hat{\psi}_{n}( \pm 1)=0 & \left(v_{n}( \pm 1)=0\right), \\
n=0: \quad \hat{\psi}_{0, y}( \pm 1)=0 & \left(u_{0}( \pm 1)=0\right) .
\end{array}\right\}
$$

The boundary condition on $v_{0}$ is automatically satisfied (since $v_{0}=(\mathrm{i} \alpha 0) \hat{\psi}_{0} \equiv 0$ everywhere). $\hat{\psi}_{0}$ is arbitrary to within a constant so we set

$$
\hat{\psi}_{0}(-1)=0
$$

This leaves one boundary condition undetermined. This corresponds to having not yet chosen the non-dimensionalizing velocity $U_{0}$, and to a resulting indeterminacy in $\hat{\psi}_{0}$. Thus the last boundary condition may be regarded as fixing the parameterization of the problem. Two reasonable choices would be either to fix the flux $(Q)$, i.e. put

$$
\left[\tilde{\Psi}_{\mathbf{P}}+\tilde{\psi}_{0}\right]_{-h}^{+h}=Q
$$

or to fix the pressure gradient $(P)$, i.e. put

$$
\left[\tilde{\Psi}_{\mathrm{P}, y y}+\hat{\psi}_{0 y y}^{x}\right]_{-h}^{+h}=\frac{2 P h}{v} .
$$

With $(8 a)$ as the last boundary condition, we set $U_{0} \equiv U_{Q}$, where $U_{Q}$ is the centreline velocity of the uniform PPF having the same flux $Q$ as our disturbed flow. Thus $\tilde{\Psi}_{\mathrm{P}}=U_{Q}\left(y-y^{3} / 3 h^{2}\right)$ and, after non-dimensionalizing by $U_{Q}$ and $h$ and using (7), (8a) becomes

Flux boundary condition: $\hat{\psi}(+1)=0$.

Similarly, using $(8 b)$ and setting $U_{0} \equiv U_{P}$, where $U_{P}$ is the centreline velocity of the uniform PPF having the same average pressure gradient $P$ as our disturbed flow, $(8 b)$ becomes

Pressure boundary condition : $\left[\tilde{\psi}_{0, y y}\right]_{-1}^{+1}=0$.

Boundary conditions $(9 a, b)$ represent the extremes of a continuous range. Solutions satisfying $(9 a)$ will have zero flux perturbation but finite pressure perturbation; as the flux perturbation $\tilde{\psi}_{0}(+1)$ is increased the pressure perturbation $\left[\tilde{\psi}_{0, y y}\right]_{-1}^{+1}$ decreases to zero where $(9 b)$ holds. 


\subsection{Flux and pressure Reynolds numbers}

Corresponding to the two different non-dimensionalizing velocities are two different Reynolds numbers, $R e_{Q}$ and $R e_{P}$. Using (8) and (9) their explicit dependence on the flux $Q$ and pressure gradient $P$ is easily determined as

and

$$
\begin{gathered}
Q=\left[\tilde{\Psi}_{\mathrm{P}}\right]_{-h}^{+h}=\frac{4 U_{Q} h}{3}, \quad R e_{Q}=\frac{h U_{Q}}{\nu}=\frac{3 Q}{4 \nu} \\
P=\frac{\nu}{2 h}\left[\tilde{\Psi}_{\mathrm{P}, y y}\right]_{-h}^{+h}=\frac{-2 \nu U_{P}}{h^{2}}, \quad R e_{P}=\frac{h U_{P}}{\nu}=\frac{-h^{3} P}{2 v^{2}} .
\end{gathered}
$$

It is important to note that the differing boundary conditions alter only the scaling of the problem, not the physies. For a given disturbed flow, there are unique perturbation stream functions to the mean flow, $\hat{\psi}_{Q 0}$ and $\hat{\psi}_{P 0}$, which differ by a multiple of the base $\mathrm{PPF}$ and the scaling factor $U_{Q} / U_{P}$. The dimensional representation of the total mean flow is

$$
\tilde{\Psi}_{\mathrm{P}}+\stackrel{\tilde{\psi}}{\psi}_{0} \equiv U_{Q}\left(y-\frac{y^{3}}{3 h^{2}}\right)+\stackrel{\tilde{\psi}}{\psi}_{Q 0}=U_{P}\left(y-\frac{y^{3}}{3 h^{2}}\right)+\stackrel{\tilde{\psi}}{\psi}_{P 0}
$$

Non-dimensionalizing by $h$ and $U_{Q}, U_{P}$ respectively:

$$
\begin{aligned}
\hat{\psi}_{P 0} & =\left(\frac{U_{Q}}{U_{P}}-1\right)\left(y-\frac{1}{3} y^{3}\right)+\left(\frac{U_{Q}}{U_{P}}\right) \hat{\psi}_{Q 0} \\
& =\left(\frac{R e_{Q}}{\operatorname{Re}_{P}}-1\right)\left(y-\frac{1}{3} y^{3}\right)+\left(\frac{\operatorname{Re}_{Q}}{\operatorname{Re}_{P}}\right) \hat{\psi}_{Q 0} .
\end{aligned}
$$

This can be used to determine the relationship between the two Reynolds numbers by substituting (11) into the boundary condition $(9 b)$

$$
\operatorname{Re}_{P}=\operatorname{Re}_{Q}\left(1-\frac{1}{4}\left[\hat{\psi}_{0 Q, y y}\right]_{-1}^{+1}\right) \text {. }
$$

These Reynolds numbers are only the same for the uniform (PPF) flow and generally $R e_{P}=f\left(R e_{Q}, \alpha\right)$ with $R e_{P}>R e_{Q}$ (Saffman 1983; Rozhdestvensky \& Simakin 1984) for the two-dimensional secondary flows we are considering. It seems that in experiments it is generally easier to fix the flux and thus $R e_{Q}$. On the other hand, most computational results have been presented in terms of the pressure Reynolds number $R e_{P}$. Hereafter, all quantities presented are non-dimensionalized by $U_{Q}$.

Equations (4)--(9) were truncated at $N=1$ Fourier modes and the resulting set of ODEs solved using the method of spectral collocation (described in $\$ 2$ ). In figure 2 we have plotted both $R e_{Q}$ and $R e_{P}$ curves for a representative $\alpha$; it is to be understood that only horizontal lines (same amplitude) correspond to the same flow (e.g. at $A=0.05, R e_{Q}=2850$ and $R e_{P}=3650$ ). The characteristic amplitude $(A)$ used here is defined as the $L_{2}$-norm of the two-dimensional secondary-flow Chebyshev coefficients for Fourier modes $n>0$.

A contradiction is reached if the Orszag \& Patera energy argument is applied to the linear stability of the two-dimensional secondary flows. If a stability transition is expected at the nose of the $R e_{Q}$ curve (as the argument suggests) then it would occur on the upper branch of the $R e_{P}$ curve; if expected at the nose of the $R e_{P}$ curve then the corresponding point would be on the lower branch of the $R e_{Q}$ curve.

If, moreover, the argument is applied to individual eigenmodes another question arises. At minimum amplitude on the two-dimensional solution curve one can expect 


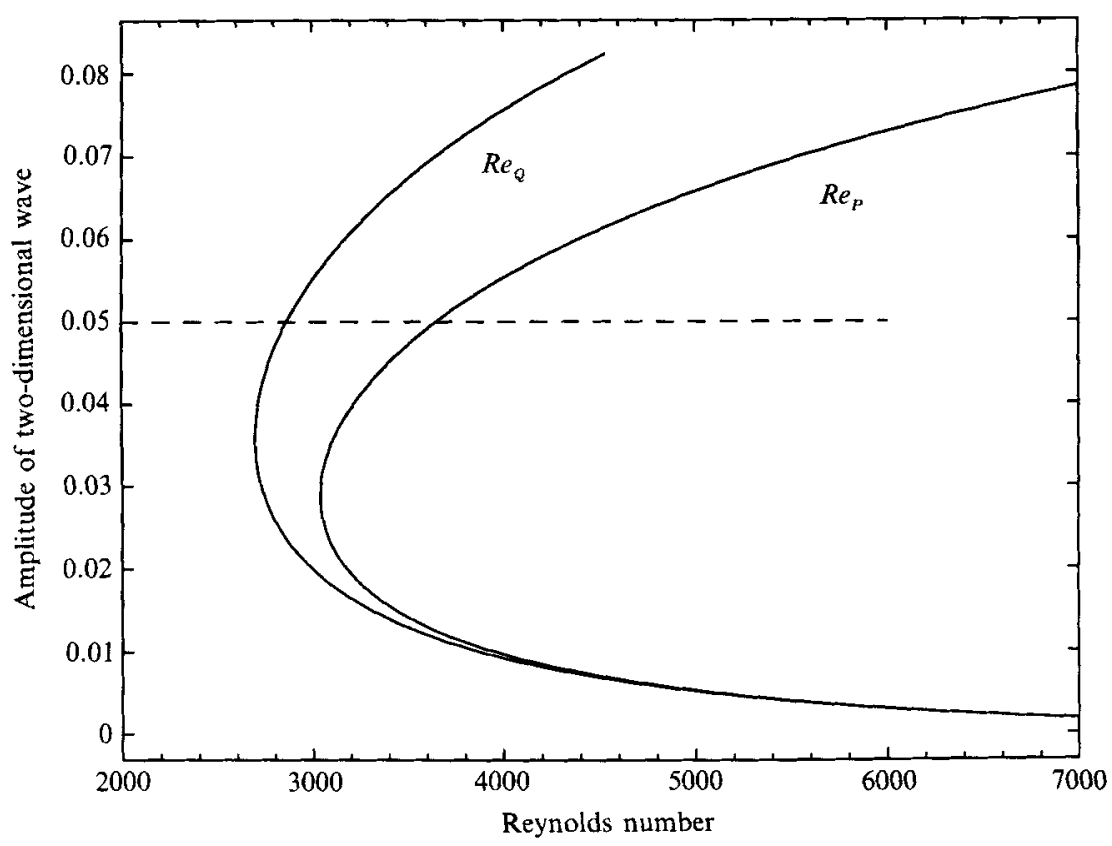

Figure 2. Comparison of pressure $\left(R e_{p}\right)$ and flux $\left(R e_{Q}\right)$ Reynolds numbers (for $\alpha=1.1$ ).

one unstable eigenvalue. The existence of a zero-growth-rate eigenvalue $(\operatorname{Re}(\sigma)=0)$ at the nose could then correspond to three situations:

(i) the unstable eigenvalue becomes stable at the nose;

(ii) another (previously stable) eigenvalue becomes unstable at the nose;

(iii) another (previously stable) eigenvalue becomes unstable on the lower branch and then goes stable at the nose.

Note that only in case (i) does one expect a stability transition.

Thus there are two problems to be considered:

To which Reynolds-number parameterization does the Orszag \& Patera argument apply (if any)?

Is the nose a stability transition $\left(\max _{\sigma} \operatorname{Re}(\sigma)=0\right.$ ), a zero-growth-rate point for an individual eigenvalue $(\operatorname{Re}(\sigma)=0)$ or a point of neutral stability $(\sigma=0)$ in the frame of reference moving with the two-dimensional secondary flow?

\section{Problem formulation}

The dimensionless form of the two-dimensional Navier-Stokes equations (1), for the streamfunction relative to a moving reference frame (speed $c)$ is

$$
\frac{\partial \nabla^{2} \psi}{\partial t}-\frac{1}{R e} \nabla^{4} \psi+\left(\psi_{y}-c\right) \nabla^{2} \psi_{x}-\psi_{x} \nabla^{2} \psi_{y}=0
$$

We look for solutions of the form

$$
\psi(x, y, t)=\Psi_{\mathrm{P}}(y)+\psi_{2 \mathrm{D}}(x, y)+\epsilon \zeta(x, y, t)
$$


where $\psi_{2 \mathrm{D}}$ describes the two-dimensional secondary flows discussed in the previous section and $\epsilon \zeta$ is an infinitesimal superharmonic disturbance. Equation (13) is separable in time, so applying the normal mode concept we write

$$
\zeta(x, y, t)=\mathrm{e}^{\sigma t} \sum_{n=-\infty}^{+\infty} \hat{\zeta}_{n}(y) \mathrm{e}^{\mathrm{i} n \alpha x}
$$

where $\operatorname{Re}(\sigma)$ is the growth rate of the disturbance and $\alpha$ is the streamwise $(x-$ direction) wavenumber of the two-dimensional secondary flow. The form (15) for the eigenfunction is a consequence of Floquet theory. Note that the eigenfunction in general contains a component with the fundamental wavenumber. Substituting (15) and the similar spectral representation (with basis functions $\hat{\Psi}$ ) for $\Psi_{2 \mathrm{D}}=\Psi_{\mathrm{P}}+\psi_{2 \mathrm{D}}$ into (13), and linearizing about the basic state $(\epsilon=0)$, we obtain the twodimensional stability equations for the $n$th Fourier mode:

$$
\begin{aligned}
-\frac{1}{R e} & \left(S_{x}^{4} \hat{\zeta}_{n}+2 S_{x}^{2} \hat{\zeta}_{n, y y}+\hat{\zeta}_{n, y y y y}\right)+S_{x}\left((U-c)\left(S_{x}^{2} \hat{\zeta}_{n}+\hat{\zeta}_{n, y y}\right)-U^{\prime \prime} \hat{\zeta}_{n}\right) \\
& +\hat{\Psi}_{y} *\left(S_{x}\left(S_{x}^{2} \hat{\zeta}+\hat{\zeta}_{y y}\right)\right)+\hat{\zeta}_{y} *\left(S_{x}\left(S_{x}^{2} \hat{\Psi}+\hat{\Psi}_{y y}\right)\right) \\
& -\left(S_{x} \hat{\Psi}\right) *\left(S_{x}^{2} \hat{\zeta}_{y}+\hat{\zeta}_{y y y}\right)-\left(S_{x} \hat{\zeta}\right) *\left(S_{x}^{2} \hat{\Psi}_{y}+\hat{\Psi}_{y y y}\right)=-\sigma\left(S_{x}^{2} \hat{\zeta}_{n}+\hat{\zeta}_{n, y y}\right)
\end{aligned}
$$

where $U(y)=1-y^{2}, y \in(-1,+1)$. We fixed the characteristic velocity as $U_{0}=U_{Q}$ (and thus $R e \equiv R e_{Q}$ ); this also affects the form of the two-dimensional flow basis functions $\hat{\Psi}$. It is straightforward to show that the eigenvalues are either real or occur in complex-conjugate pairs. The accompanying boundary conditions are

$$
\left.\begin{array}{rlrl}
n \neq 0: & \hat{\zeta}_{n, y}( \pm 1)=0 & \left(u_{n}( \pm 1)=0\right), \\
\hat{\zeta}_{n}( \pm 1)=0 & \left(v_{n}( \pm 1)=0\right), \\
n=0: & \hat{\zeta}_{0, y}( \pm 1)=0 & \left(u_{0}( \pm 1)=0\right) .
\end{array}\right\}
$$

The boundary condition on $v_{0}$ is again automatically satisfied. $\hat{\zeta}_{0}$ is arbitrary to within a constant so we set

$$
\hat{\zeta}_{0}(-1)=0 \text {. }
$$

Equations (16)-(18) describe an infinite set of two-dimensional disturbances, the form of which is specified by the final boundary condition. Two reasonable choices might be

or

$$
\begin{aligned}
\hat{\zeta}_{0}(+1)-\hat{\zeta}_{0}(-1)=0 & \text { (constant flux) } \\
\hat{\zeta}_{0, y y}(+1)-\hat{\zeta}_{0, y y}(-1)=0 & \text { (constant pressure) }
\end{aligned}
$$

Equations $(19 a, b)$ are only two of many possibilities for boundary conditions. One can look for solutions of a particular $y$-symmetry; both antisymmetric $\left(\hat{\zeta}_{n}(-y)=(-1)^{n+1} \hat{\zeta}_{n}(+y)\right)$ and symmetric $\left(\hat{\zeta}_{n}(-y)=(-1)^{n} \hat{\zeta}_{n}(+y)\right)$ disturbances satisfy the equations. In fact all computed eigensolutions appear to be of one of these two forms for constant-flux disturbances. Note that $(19 a)$ and $(19 b)$ are both satisfied identically for symmetric disturbances.

To solve the set of ODEs and boundary conditions in (16)-(19) we applied the technique of spectral collocation and wrote

$$
\hat{\zeta}_{n}(y)=\sum_{k=0}^{K} a_{n k} T_{k}(y)
$$


where $T_{k}(y)$ is the $k$ th Chebyshev polynomial. Evaluating the resulting equations at the maxima of the $K$ th Chebyshev polynomial (as suggested in Orszag $\&$ Gottlieb 1977) yielded a (discrete) generalized eigenvalue problem of the form $G a=\sigma B a$ for the complex eigenvalue $\sigma$ and the associated eigenvector $a$. This was solved using standard numerical methods.

We note in passing that a weakly nonlinear analysis would be inappropriate in this stability problem because we are interested in the stability behaviour of finite- (generally large) amplitude steady states rather than the small amplitudes appropriate in the weakly nonlinear theory. Weakly nonlinear analysis of behaviour near the nose of the Orr-Sommerfeld curve for the stability of PPF is a completely different problem than the analysis of the stability of secondary flows near the nose of the amplitude - Reynolds-number solution curve. Indeed, most of the numerical computations (see $\$ 4$ ) are for wavenumbers $\alpha$ such that PPF is linearly stable for all Reynolds numbers, and there is no weakly nonlinear theory.

\section{Eigensolutions with $\sigma=0$}

\subsection{Phase-shift solution}

If we substitute $\psi \equiv \Psi_{2 \mathrm{D}}$ (as defined in $\S 2$ ) into (13) the nonlinear equation for the two-dimensional secondary flow can be written

$$
-\frac{1}{R e} \nabla^{4} \Psi_{2 \mathrm{D}}+\left(\Psi_{2 \mathrm{D}, y}-c\right) \nabla^{2} \Psi_{2 \mathrm{D}, x}-\Psi_{2 \mathrm{D}, x} \nabla^{2} \Psi_{2 \mathrm{D}, y}=0
$$

which is of the form

$$
N\left(\Psi_{2 \mathrm{D}}(x, y), R e, \alpha, c\right)=0,
$$

and we can derive the two-dimensional stability equations (16) in a formal manner. A general unsteady solution $\Psi_{2 \mathrm{D}}(x, y)+\epsilon \mathrm{e}^{\alpha t} \zeta(x, y)$ satisfies

$$
\left.\frac{\partial}{\partial \epsilon}\right|_{\epsilon=0} N\left(\Psi_{2 \mathrm{D}}(x, y)+\epsilon \mathrm{e}^{\sigma t} \zeta(x, y), R e, \alpha, c\right)=\frac{\partial \nabla^{2}\left(\mathrm{e}^{\sigma t} \zeta(x, y)\right)}{\partial t},
$$

whence

$$
\frac{\delta N}{\delta \Psi} \zeta=\sigma \nabla^{2} \zeta
$$

Also, differentiating (21) in the $x$-direction:

$$
\frac{\delta N \partial \Psi}{\delta \Psi \partial x}=0
$$

and comparing (23) and (24) shows that the eigenvalue $\sigma=0$ is always a solution of the stability equations, with associated eigenfunction $\zeta_{\mathrm{ps}}=\partial \Psi / \partial x$. (We note that this also satisfies the boundary conditions on $\zeta$, regardless of the choice of boundary condition for mode $n=0$.) We can call this the phase-shift solution, since it represents the trivial two-dimensional 'disturbance' caused by shifting a known twodimensional secondary flow along the $x$-axis.

\subsection{Neutral stability at the "nose'}

We want to show now that there is an additional zero eigenvalue at the nose of the appropriate $R e$ curve; on the $R e_{P}$ curve for constant-pressure $(P)$ disturbances and on the $R e_{Q}$ curve for constant-flux $(Q)$ disturbances. Furthermore, the eigenfunction corresponding to this zero eigenvalue is also the phase-shift eigenfunction ; that is, at 
the nose there is a zero eigenvalue of algebraic multiplicity 2 and geometric multiplicity 1 (which we will denote for brevity as AM 2, GM 1) so that the null space is one-dimensional. For a discussion of these terms, see Gollub \& Van Loan (1983).

Consider (21), at some fixed $\alpha$, parameterized by some arclength $s$ :

$$
N(\Psi, R e, c ; s)=0,
$$

where $\Psi \equiv \Psi_{2 \mathrm{D}}$ for convenience. Differentiating this along the solution curve gives

$$
\begin{aligned}
\frac{\mathrm{d} N}{\mathrm{~d} s} & \equiv \frac{\delta N}{\delta \Psi} \frac{\mathrm{d} \Psi}{\mathrm{d} s}+\frac{\partial N}{\partial R e} \frac{\mathrm{d} R e}{\mathrm{~d} s}+\frac{\partial N}{\partial c} \frac{\mathrm{d} c}{\mathrm{~d} s} \\
& =N_{\Psi} \dot{\Psi}+N_{R e} \dot{R} e+N_{c} \dot{c}=0 .
\end{aligned}
$$

At the limit point ('nose') in $R e, \dot{R} e=0$, whence

$$
N_{\psi} \dot{\Psi}=-\dot{c} N_{c} \text {. }
$$

If $\dot{c}=0$, then $\sigma=0$ is a second eigenvalue of the stability problem (23), with associated eigenfunction $\zeta=\dot{\Psi}$ ' (the 'tangent vector'), where $\dot{\Psi}$ satisfies appropriate boundary conditions. In this case we have AM 2 and GM 2 (i.e. index one).

But in general $\dot{c} \neq 0$, and then $\dot{\Psi} / \dot{c}$ is a generalized eigenfunction for the phaseshift eigenfunction $\zeta_{\mathrm{ps}}$. To see this, note that from (20),

$$
N_{c} \equiv-\nabla^{2} \frac{\partial}{\partial x} \Psi=-\nabla^{2} \zeta_{\mathrm{ps}}
$$

whence (26) implies

$$
N_{\psi} \phi=\nabla^{2} \zeta_{\mathrm{ps}}
$$

where $\phi=\dot{\Psi} / \dot{c}$ satisfies the same boundary conditions as $\psi$ (because $\dot{\Psi}=\dot{\Psi}_{\mathrm{P}}+\dot{\psi}=\dot{\psi}$ on the walls). That is, on the $R e_{Q}$ curve

$$
\hat{\phi}_{n}( \pm 1)=0
$$

and on the $R e_{P}$ curve

$$
\left.\begin{array}{rr}
n>0: & \hat{\phi}_{n}( \pm 1)=\hat{\phi}_{n}^{\prime}( \pm 1)=0, \\
n=0: & \hat{\phi}_{0}(-1)=\left[\hat{\phi}_{0}^{\prime \prime}\right]_{-1}^{+1}=\hat{\phi}_{0}^{\prime}( \pm 1)=0,
\end{array}\right\}
$$

where $\hat{\phi}_{n}(y)=\hat{\psi}_{n} / \dot{c}$. Suppose now we have a generalized eigenvalue problem, $S \xi=\sigma T \xi$, with $\sigma=0$ an eigenvalue of GM 1 and AM 2. Then it can be shown (using the Jordan Normal Form of $S$ and calculating the eigenvectors explicitly) that the existence of a zero eigenvalue of GM 1 and AM 2 or more is equivalent to the existence of an eigenfunction $\eta$ and generalized eigenfunction $y$ such that

$$
\begin{aligned}
s \eta & =0, \\
s y-T \eta & =0 .
\end{aligned}
$$

In the context of the eigenvalue problem (23), we have $S \equiv N_{\psi}, T \equiv \nabla^{2}$ and $\eta \equiv \zeta_{\text {ps }}$, whence (29) and (30) become (24) and (27) with $\phi \equiv y$. Thus there is an eigenvalue $\sigma=0$ of GM 1 and at least AM 2 at the nose $\dot{R} e=0$ (because (27) applies only at the nose).

To which Re this corresponds depends on the particular stability problem being solved. On the $R e_{Q}$ curve, $\psi$ satisfies $(6),(7)$ and $(9 a)$, and thus $(28 a)$ is satisfied. By $(19 a)$, these are just the conditions for the $Q$-stability problem. A similar argument 
applies to the $R e_{P}$ curve. In summary we find that constant-flux disturbances have a zero eigenvalue (with algebraic multiplicity 2 and geometric multiplicity 1 ) at the nose of the $R e_{Q}$ curve, and constant-pressure disturbances have a zero eigenvalue (i.e. neutral stability) at the nose of the $R e_{P}$ curve. The associated eigenfunction is the phase-shift solution $\zeta_{\text {ps }}$.

The contradictory conclusions reached by applying the Orszag \& Patera energy argument $(\$ 1.2)$ can thus be reconciled if one accounts for a non-uniqueness in the form of the two-dimensional disturbance considered in the stability problem; it has to be of a form appropriate to the particular Reynolds-number parameterization.

\section{Numerical results}

The above results are illustrated for two-dimensional stability at various points along the solution curve for $\alpha=1.1$. The curve appears to bifurcate from infinity for this wavenumber. Calculations show that the behaviour at $\alpha=1$ (where the curve bifurcates from $R e_{Q} \approx 5800$ ) is similar to that shown here. The two-dimensional secondary flows were computed with $N=1$ Fourier modes in the $x$-direction and $K=50$ Chebyshev modes in the $y$-direction. The two-dimensional stability was computed with $N=1, K=32$ and $N=1, K=50$ (at high Reynolds numbers). The resolution in $x$ is insufficient to give more than qualitative results. A number of calculations with $N=2$ (for both secondary flow and stability) indicate that although the positions of the bifurcation points change from $N=1$ to $N=2$, the nature of the bifurcation and $\operatorname{Im}(\sigma)$ at the bifurcation point remain the same. The secondary flows and stability calculations were performed on a VAX-11/750; calculation of all the eigenvalues of the stability problem for one secondary flow took about 10 minutes CPU time for $N=1, K=32$ and 40 minutes for $N=2, K=32$. The secondary flows themselves took 1-5 minutes and 5-30 minutes for $N=1, K=50$ and $N=2, K=50$ respectively, the exact time depending on the number of Newton iterations necessary.

The major restriction on performing computations with larger $N$ is not just computing time, but rather memory limitations of the machines available. It has been shown (Herbery 1976) that $N=2$ generally is sufficient to resolve the steady state and we have found that the qualitative behaviour of the eigenvalue problem is preserved when going from $N=1$ to $N=2$. Refinement of the exact numerical values seems less important, for this flow, than the more interesting and possibly more universal qualitative features of the two-dimensional instability.

We first consider constant-flux disturbances (' $Q$-stability') (boundary condition $(19 a))$ and plot the maximum growth rate $(\max R e(\sigma))$ in figure 3 . The maximum eigenvalue is purely real in the Reynolds-number range shown. At the nose of the $R e_{Q}$ curve this eigenvalue passes through zero and consequently there is a stability transition. However on the $R e_{P}$ curve this stability transition occurs on the 'upper branch', after the nose is reached. Of course the actual flow where this stability transition occurs (and the amplitude of that flow) is the same. Although a stability transition occurs at the $R e_{Q}$ nose, the upper branch does not remain stable. As is shown in figure 4 , a different eigenvalue becomes unstable at $R e_{Q}=6300$. Because this eigenvalue has $\operatorname{Im}(\sigma) \neq 0$ at the zero-growth-rate point $(\operatorname{Re}(\sigma)=0)$, a Hopf bifurcation to a family of travelling-wave solution occurs. Combined with the underlying steady travelling wave, this is a bifurcation to a spatially periodic flow with two frequencies (quasi-periodic) in time. Jiménez (1987) has used an unsteady code for $\alpha=1$ to follow this bifurcation and has found quasi-periodic flows, period 


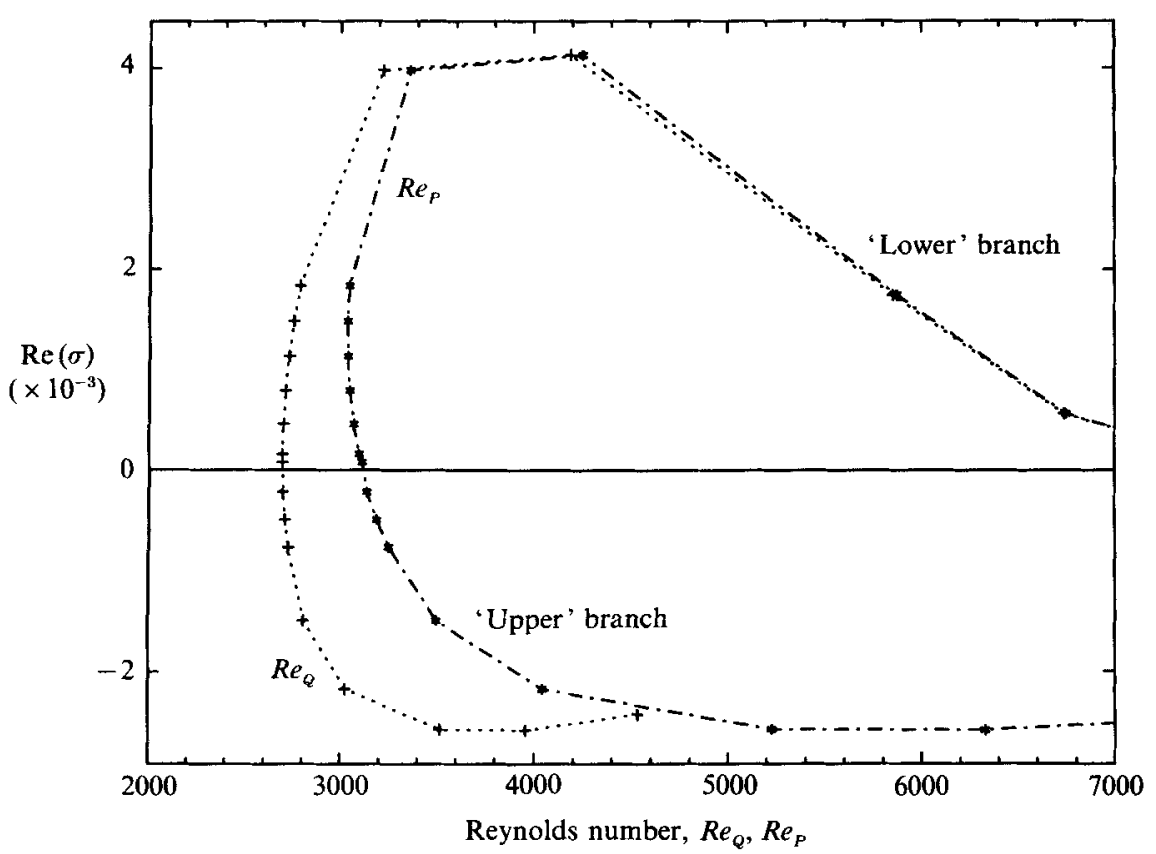

Figure 3. Maximum growth rate $(\max \operatorname{Re}(\sigma))$ for two-dimensional $Q$-stability, for $\alpha=1$.1. The most unstable eigenvalue is always purely real $(\operatorname{Im}(\sigma)=0)$ in this case.
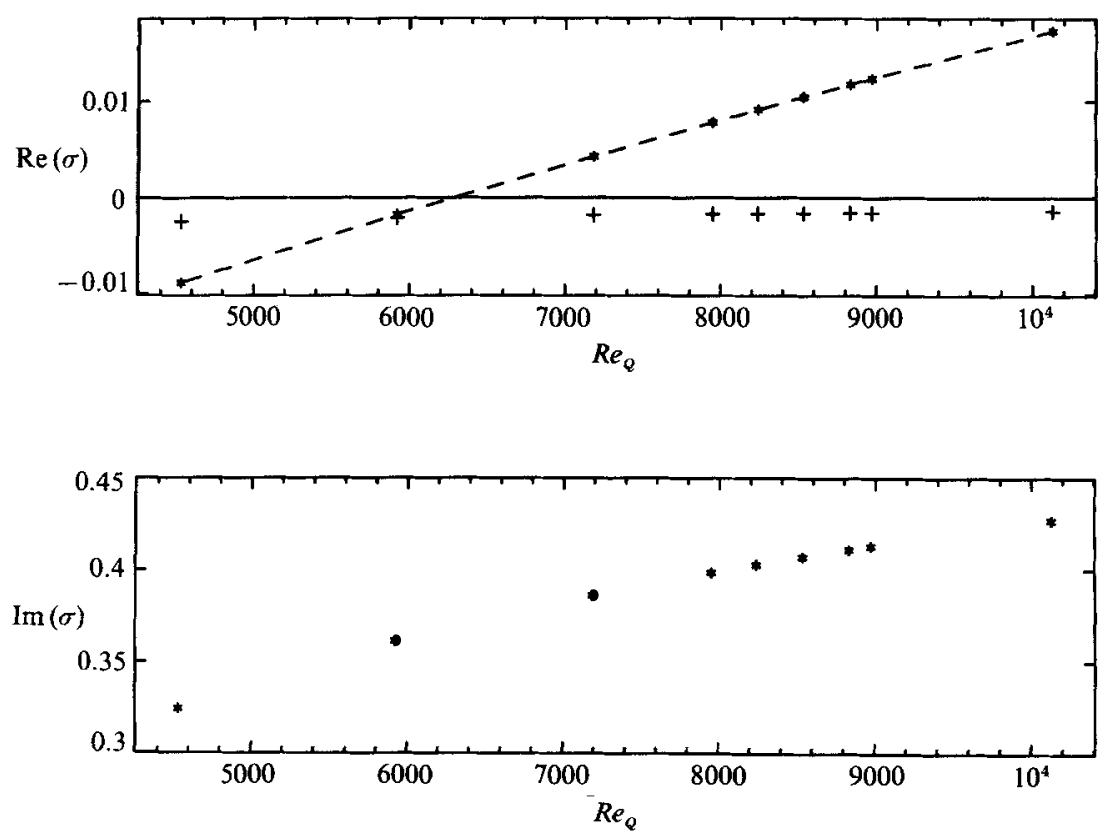

Figure 4. Stability transition for $Q$-disturbances, on the upper branch, for $\alpha=1.1$. This is a continuation of figure 3 ; the most unstable (real) eigenvalue at $R e_{Q}=4530(+$ in both figures) is overtaken by another (complex) eigenvalue $(*)$ at $R e_{Q}=5920$. 


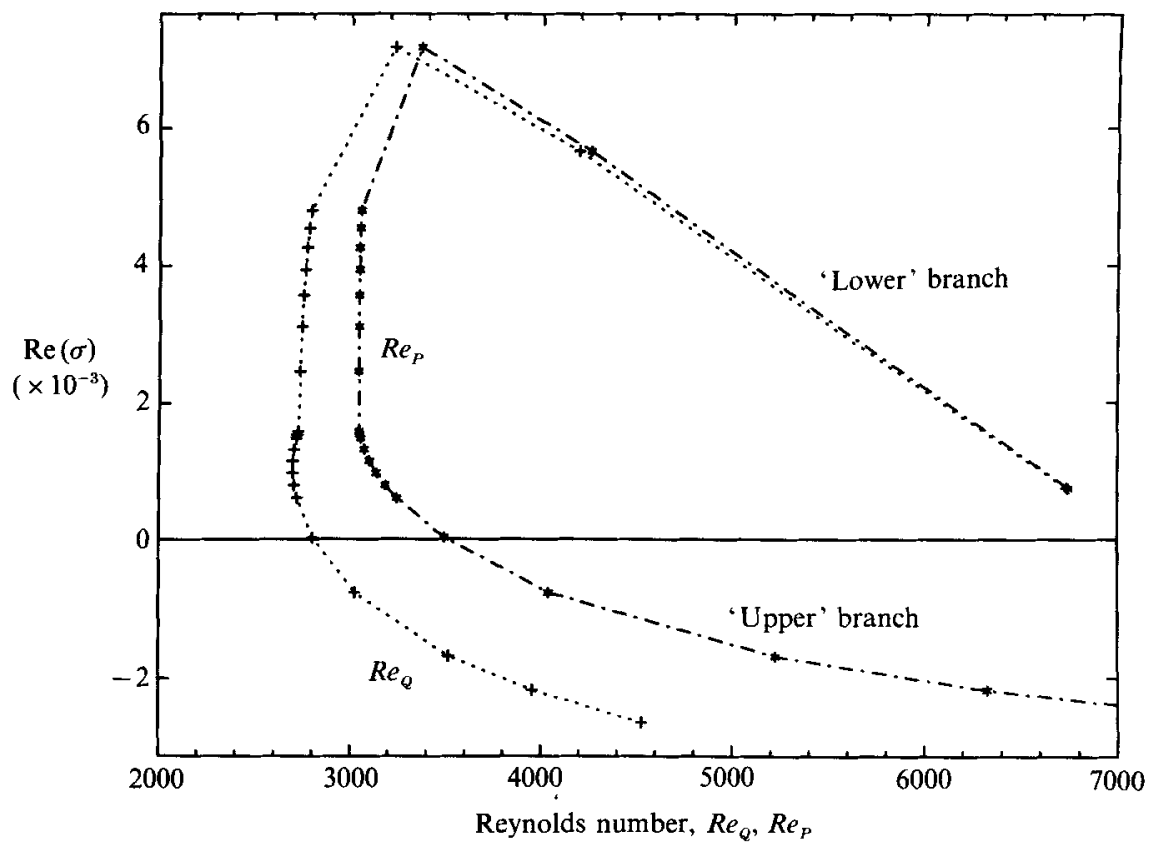

Figure 5. Maximum growth rate $(\operatorname{Re}(\sigma))$ for two-dimensional $P$-stability, for $\alpha=1.1$.
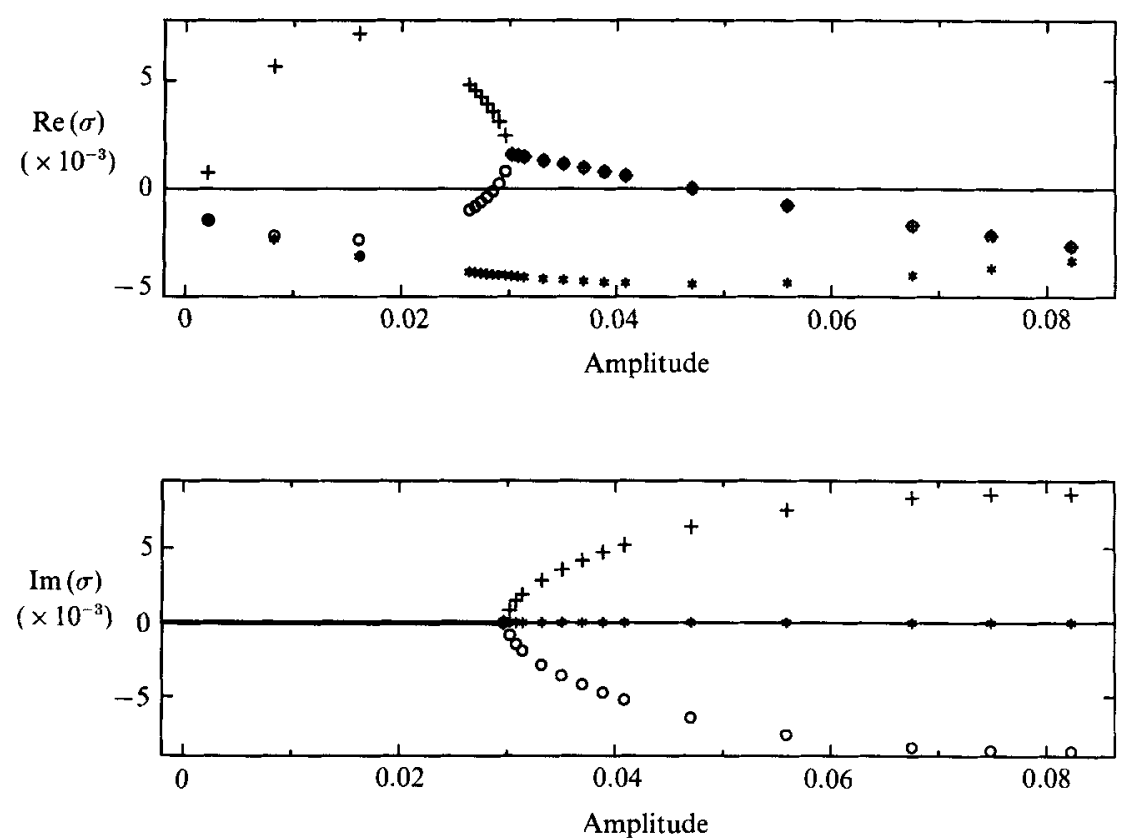

FIGURE 6. Stability transition for $P$-disturbances, on the upper branch, for $\alpha=1$.1. The nose occurs at $A=0.028, R e_{P}=3041$ and the bifurcation at $A=0.047, R e_{P}=3500 . R e_{Q}=2800$. The heavy line for $A<0.028$ indicates that $\operatorname{Im}(\sigma)=0$ for the eigenvalues shown. 
doublings and evidence of chaos. The Reynolds number increases along the stable branch of quasi-periodic solutions, as is expected for a supercritical Hopf bifurcation. The Reynolds numbers at which the Hopf bifurcation occurs calculated by Jiménez agree well with those found using our method, confirming the belief that the qualitative behaviour is well described by the results with the $N=1$ truncation.

Turning now to constant-pressure disturbances ('P-stability') (boundary condition (19b)), we might expect a similar behaviour; a stability transition at the $R e_{P}$ nose and at some point on the lower branch of the $R e_{Q}$ curve (because the nose of the $R e_{Q}$ curve occurs at higher amplitude than that of the $R e_{P}$ curve). At least at $\alpha=1.1$, this does not occur. As figure 5 illustrates, the maximum eigenvalue remains unstable on the lower branch and part of the upper branch of the $R e_{P}$ curve. Thus no stability transition occurs at the $R e_{P}$ nose.

As expected from $\S 3$, however, there is a neutrally stable eigenvalue at the nose of the $R e_{p}$ curve. Figure 6 plots the three most unstable eigenvalues. At low amplitude there is only one (real) unstable eigenvalue. At the nose there is a zero eigenvalue as predicted, but it is that of another mode becoming unstable. On the upper branch these two unstable real modes coalesce to form a complex-conjugate pair with $\operatorname{Re}(\sigma) \rightarrow 0$ at $A=0.047$, where $R e_{P}=3500$ (the nose occurs at $A=0.028$, $\left.R e_{P}=3041\right)$. Since $\operatorname{Im}(\sigma) \neq 0$ at this point, this is a bifurcation to a quasi-periodic flow, but at a significantly lower Reynolds number $\left(R e_{Q}=2800\right.$ at the bifurcation point) than for constant-flux disturbances. Thus the upper branch becomes stable to $\boldsymbol{P}$-disturbances with increasing Reynolds number. Since the theory of Hopf bifurcations (Marsden \& McCracken 1976) predicts that a subcritical bifurcation from this point would be stable, this raises the possibility of this bifurcation leading to stable quasi-periodic flows at lower Reynolds numbers (possibly smaller than the minimum for two-dimensional secondary flows).

Note that the principal eigenvalues plotted in figures 36 are associated with asymmetric or antisymmetric eigensolutions. If, on the other hand the maximum eigenvalues corresponded to symmetric eigensolutions, satisfying both flux and pressure boundary conditions identically (\$2), there would be no difference in the stability behaviour of constant-flux and constant-pressure disturbances.

\section{Summary}

We have examined the two-dimensional superharmonic stability of twodimensional secondary flows in plane Poiseuille flow. Previously simplistic energy arguments have been used to predict (incorrectly) a stability transition at the 'nose' of the two-dimensional solution curve. A neutrally stable eigenvalue $(\sigma=0)$ does occur at the 'nose' of the two-dimensional curve, at least if one considers twodimensional disturbances of a form appropriate to the particular curve Reynolds number. However, this does not necessarily correspond to a change from unstable to stable for this eigenvalue as the amplitude increases, for the change can be from stable to unstable. Nor is there necessarily a stability transition at the nose because other eigenvalues may remain unstable at this point.

It was found that the neutrally stable eigenvalue at the nose is of algebraic multiplicity 2 and geometric multiplicity 1, with the corresponding eigenfunction just the phase-shift solution. The proof of this is general, and should apply to other systems where the stability of travelling waves to travelling disturbances of the form (15) is considered. Numerical evidence of the above assertions is given for a streamwise wavenumber of $\alpha=1.1$. Eigenvalues with zero growth rate $(\operatorname{Re}(\sigma)=0)$ 
were found on the upper branch of the two-dimensional secondary flow eurve for both constant flux and constant-pressure disturbances; because $\operatorname{Im}(\sigma) \neq 0$ when $\operatorname{Re}(\sigma)=0$, these are bifurcations to quasi-periodic flows.

We have not investigated the stability behaviour at higher Reynolds numbers to ascertain whether the upper branch restabilizes to $Q$-disturbances or destabilizes to $P$-disturbances. Nor have we followed the quasi-periodic flows shed at the bifurcation points. Nevertheless, the stability behaviour of the two-dimensional secondary flows and the nature of the Hopf bifurcations give a much richer picture of possible instabilities instead of the accepted one of a simple stability transition at the nose of the two-dimensional secondary flow curve.

We wish to acknowledge a number of helpful discussions with Steve Wiggins and Michael Landman. This work was supported by the Office of Naval Research (Grant N00014-85-K-0205) and the Department of Energy, Office of Energy Sciences (DE-AS03-76ER-72012), Applied Mathematical Sciences (KC-07-01-01).

\section{REFERENCES}

Gollu B, G. H. \& VAn Loan, C. F. 1983 Matrix Computations. Johns Hopkins.

Herbert, Th. 1976 Periodic secondary motions in a plane channel. In Proc. 5th Intl Conf. on Numerical Methods in Fluid Dynamics. (ed. A. I. van de Vooren \& P. J. Zandberger). Lecture Notes in Physics, vol. 59, pp. 235-240. Springer.

Jiménfz, J. 1987 Bifurcations and bursting in two-dimensional Poiseuille flow. Phys. Fluids 30, 3644-3646.

Marsden, J. E. \& McCracken, M. (ED.) 1976 The Hopf Bifurcation and Its Applications. Springer.

Milinazzo, F. A. \& Saffman, P. G. 1985 Finite-amplitude waves in plane viscous shear flows. J. Fluid Mech. 160, 281-295.

Orszag, S. A. \& GotTlieb, D. 1977 Numerical Analysis of Spectral Methods: Theory and Applications, p. 14. Society for Industry and Applied Mathematics, vol. 24.

Orszag, S. A. \& Patera, A. T. 1981 Subcritical transition to turbulence in planar shear flows. In Transition and Turbulence (ed. R. E. Meyer), pp. 127-146. Academic.

Orszag, S. A. \& Patera, A. T. 1983 Secondary instability of wall-bounded shear flows. J. Fluid Mech. 128, 347-353.

Rozhdestvensky, B. L. \& Stmakin, I. N. 1984 Secondary flows in a plane channel: their relationship and comparison with turbulent flows. J. Fluid Mech. 147, 261-289.

Saffman, P. G. 1983 Vortices, stability, and turbulence. Ann. N.Y. Acad. Sci. 404, 12-24.

Zahn, J.-P., Toomre, J., Spiegel, E. A. \& Gough, D. O. 1974 Nonlinear cellular motions in Poiseuille channel flow. J. Fluid Mech. 64, 319-345. 